\title{
Clinical Implications in Testicular Cancer
}

\author{
Gerasimos J. Alivizatos and Pavlos A. Pavlakis
}

\subsection{Diagnosis: Staging}

Diagnosis of testicular tumors is performed with ultrasonography (US). MRI of the scrotum has higher sensitivity and specificity than US but should be used in ambiguous cases only. Nodal metastases in the abdomen and in the thorax are performed mainly with CT scans. MRI scans have been used as well and can be helpful in some cases (ambiguous CT findings, allergy to contrast materials), but its wide use in the staging procedure is not justified. PET scans are also not recommended in the staging procedure of testicular cancer [1].

\subsection{Follow-Up Strategies}

Follow-up after treatment is being performed with tumor markers and imaging modalities. CT scans are used to evaluate lymph nodes and PET scans are used as well. The main role of
FDG-PET, however, lies in the characterization of postchemotherapy residual masses, due to its ability to differentiate between necrosis/fibrosis and residual or recurrent viable tumor. In this context, PET-CT study is recommended in the postchemotherapy management of pure seminoma patients in order to assess whether residual viable tumor is present. In nonseminomatous germ cell tumors of the testis, however, FDG-PET should be used with caution, as it cannot differentiate between mature teratoma and fibrosis [2].

\section{References}

1. Winter C, Pfister D, Busch J et al (2012) Residual tumor size and IGCCCG risk classification predict additional vascular procedures in patients with germ cell tumors and residual tumor resection: a multicenter analysis of the German Testicular Cancer Study Group. Eur Urol 61(2):403-409

2. Daneshmand S, Albers P, Fossa A et al (2012) Contemporary management of post chemotherapy testis cancer. Eur Urol 62(5):867-876

G. J. Alivizatos $(\bowtie) \cdot$ P. A. Pavlakis

3rd Urological Department, Hygeia Hospital,

Athens, Greece

e-mail: gali@hol.gr 